\title{
Biohybrid Thin Films for Measuring Contractility in Engineered Cardiovascular Muscle
}

\author{
Patrick W. Alford, PhD*, Adam W. Feinberg, PhD*, Sean P. Sheehy, ALM, and Kevin Kit Parker, \\ PhD \\ Disease Biophysics Group, Harvard Stem Cell Institute, Wyss Institute for Biologically Inspired \\ Engineering, School of Engineering and Applied Sciences, Harvard University, Cambridge, MA \\ 02138
}

\begin{abstract}
In vitro cardiovascular disease models need to recapitulate tissue-scale function in order to provide in vivo relevance. We have developed a new method for measuring the contractility of engineered cardiovascular smooth and striated muscle in vitro during electrical and pharmacological stimulation. We present a growth theory-based finite elasticity analysis for calculating the contractile stresses of a 2D anisotropic muscle tissue cultured on a flexible synthetic polymer thin film. Cardiac muscle engineered with neonatal rat ventricular myocytes and paced at $0.5 \mathrm{~Hz}$ generated stresses of $9.2 \pm$ $3.5 \mathrm{kPa}$ at peak systole, similar to measurements of the contractility of papillary muscle from adult rats. Vascular tissue engineered with human umbilical arterial smooth muscle cells maintained a basal contractile tone of $13.1 \pm 2.1 \mathrm{kPa}$ and generated another $5.1 \pm 0.8 \mathrm{kPa}$ when stimulated with endothelin-1. These data suggest that this method may be useful in assessing the efficacy and safety of pharmacological agents on cardiovascular tissue.
\end{abstract}

\section{Introduction}

Muscle in the cardiovascular system pumps blood and regulates vascular resistance, qualifying the contractile function of these tissues critically important in development and disease. Currently however, there is no simple and repeatable method for measuring functional mechanical output of cardiovascular (CV) muscle cells in vitro that can be compared with traditional biochemical assays of protein and gene expression. Tissue engineered CV muscle provides a high fidelity means of studying their diseases and potential therapeutic interventions. Recent reports demonstrate the ability to organize muscle cells into CV tissues that recapitulate a broad range of in vivo functions. Kleber and colleagues [1-2] first developed patterned cell cultures of ventricular myocytes to study the physics of excitation wavefront propagation through custom designed microenvironments. These studies were the first attempts to recapitulate the ventricular microarchitecture in vitro. Later, the application of soft lithography techniques to building 2D anisotropic monolayers of ventricular myocytes by Tung and

(C) 2009 Elsevier Ltd. All rights reserved.

Corresponding author: Kevin Kit Parker 29 Oxford St (Rm 322A) Cambridge, MA 02138 Phone: (617) 495-2850 Fax: (617) 495-9837 kkparker@ seas.harvard.edu.

Authors contributed equally to this work

Publisher's Disclaimer: This is a PDF file of an unedited manuscript that has been accepted for publication. As a service to our customers we are providing this early version of the manuscript. The manuscript will undergo copyediting, typesetting, and review of the resulting proof before it is published in its final citable form. Please note that during the production process errors may be discovered which could affect the content, and all legal disclaimers that apply to the journal pertain.

Disclosures

None 
colleagues [3-4] allowed for higher fidelity studies of action potential propagation. Comparable approaches, such as growing ventricular myocytes on grooved substrata, have allowed for studies on calcium metabolism in anisotropic cultures [5]. Similarly, vascular smooth muscle cells have been patterned into fibrils and aligned monolayers using grooved substrates [6-7] to control muscular alignment and differentiation. However, efforts to adapt the same culture methods to measure contractility have yet to emerge due to isometric constraint to a rigid or semi-rigid substrate. Three dimensional cardiac [8-9] and vascular [10-12] tissue engineering techniques enable stress measurement, but require physical attachment to force transducers and cannot direct microscale aligned tissue structure. Thus, we sought to combine the repeatable structures of previous 2-D techniques with the stress measuring capabilities of recent 3-D systems.

Previously, we reported on a muscular thin film (MTF) technique that enables cardiac muscle monolayers, engineered on a thin, flexible 2-D substrate, to undergo 3-D deformation [13]. The MTF is a bilaminate of polydimethylsiloxane (PDMS) and a cell monolayer, whose stress state is indicated by its radius of curvature. To determine the stress in the cell layer, we have developed a new elasticity based analysis that is more accurate and robust than previous analyses [13-15], enabling concurrent measurement of the orthotropic contraction stress in the muscle and the stress-free shortening that the muscle would undergo if unconstrained.

Additionally, we build on the MTF technique by expanding to vascular smooth muscle to create microstructured $\mathrm{CV}$ tissues in vitro and directly measure stress generation due to electrical and pharmacological stimulation.

\section{Methods}

\subsection{Micropatterned Substrate and Muscular Thin Film Fabrication}

Muscular thin films (MTFs) were fabricated via a multi-step spin coating process [13]. Briefly, poly(N-isopropylacrylamide) (PIPAAm, Polysciences, Inc.) was dissolved in butanol and spin coated onto $25 \mathrm{~mm}$ diameter glass cover slips. Sylgard 184 (Dow Corning)

polydimethylsiloxane (PDMS) elastomer was spin coated on top of the PIPAAm, then cured at $65^{\circ} \mathrm{C}$ for 4 hours. In some cases, the PDMS was doped with $0.2 \mu \mathrm{m}$ fluorospheres at a concentration $<0.01 \%$ by volume. Every third sample was retained for subsequent thickness measurement of the PDMS layer using a stylus profilometer (Dektak 6M, Veeco Instruments Inc.). Microcontact printing of extracellular matrix $(\mathrm{ECM})$ proteins was used to spatially encode guidance cues that direct the self-assembly of chemically disassociated muscle cells into tissue monolayers. ECM proteins fibronectin (FN) or laminin (LAM) were micropatterned onto the PDMS as lines. To micropattern ECM, PDMS stamps with uniformly spaced ridges (10 $\mu \mathrm{m}$ wide ridges spaced by $10 \mu \mathrm{m}$, or $20 \mu \mathrm{m}$ wide with $20 \mu \mathrm{m}$ spaces) were used to transfer ECM to the PDMS surface creating ECM lines of according size. For anisotropic cardiac myocyte patterns, $2.5 \mu \mathrm{g} / \mathrm{mL}$ of FN in DI water was incubated on the cover slip surface for 15 minutes. For the immunostained samples to assess cytoskeletal structure, PDMS coated cover slips were fabricated using the identical process except the layer of PIPAAm was omitted.

\subsection{Cardiomyocyte harvest, seeding and culture}

Neonatal rat ventricular myocytes were isolated from 2-day old neonatal Sprague-Dawley rats based on published methods [16]. Briefly, ventricles were extracted and homogenized by washing in Hanks balanced salt solution followed by digestion with trypsin and collagenase with agitation overnight at $4^{\circ} \mathrm{C}$. Cells were re-suspended in M199 culture medium supplemented with $10 \%$ (v/v) heat-inactivated fetal bovine serum (FBS), $10 \mathrm{mM}$ HEPES, 3.5 $\mathrm{g} / \mathrm{L}$ glucose, $2 \mathrm{mM}$ L-glutamine, $2 \mathrm{mg} / \mathrm{L}$ vitamin B-12, and $50 \mathrm{U} / \mathrm{ml}$ penicillin and seeded on anisotropically patterned FN at a density of 1 million cells per cover slip. Samples were incubated under standard conditions at $37^{\circ} \mathrm{C}$ and $5 \% \mathrm{CO}_{2}$. Media was exchanged with 
maintenance media (2\% FBS) every 48 hours until use. The MTFs were cultured for a period of 4 to 6 days and then used in the contractility assay. All procedures were approved by Harvard animal care and use committee.

\subsection{Vascular Smooth Muscle Cell Seeding and Culture}

Human umbilical artery vascular smooth muscle cells (VSMCs) in passage 3 (Lonza) were cultured in growth medium, of M199 culture medium supplemented with $10 \%$ FBS, $10 \mathrm{mM}$ HEPES, $3.5 \mathrm{~g} / \mathrm{L}$ glucose, $2 \mathrm{mM}$ L-glutamine, $2 \mathrm{mg} / \mathrm{L}$ vitamin B-12, $50 \mathrm{U} / \mathrm{ml}$ penicillin and 50 $\mathrm{U} / \mathrm{ml}$ streptomycin. Passages 5-7 were used for all experiments. VSMCs were seeded on MTFs on a 10×10 LAM line pattern at $25,000 \mathrm{cells} / \mathrm{cm}^{2}$. After seeding, samples were incubated in growth medium for 48 hours and then for an additional 48 hours in serum free medium to induce a contractile phenotype [17].

\subsection{Muscular Thin Film Contractility Assay}

To release the MTFs, cover slips were removed from the incubator and transferred to a Petri dish filled with $37^{\circ} \mathrm{C}$ normal Tyrode's solution (1.192 $\mathrm{g}$ of HEPES, $0.901 \mathrm{~g}$ of glucose, 0.265 $\mathrm{g}$ of $\mathrm{CaCl}_{2}, 0.203 \mathrm{~g}$ of $\mathrm{MgCl}_{2}, 0.403 \mathrm{~g}$ of $\mathrm{KCl}, 7.889 \mathrm{~g}$ of $\mathrm{NaCl}$ and $0.040 \mathrm{~g}$ of $\mathrm{NaH}_{2} \mathrm{PO}_{4}$ per liter of deionized water). The Petri dish was placed on a stereomicroscope (Model MZ6 with darkfield base, Leica Microsystems Inc.) and rectangles $\sim 2.5 \mathrm{~mm}$ wide and $7 \mathrm{~mm}$ long were cut out using a straight-blade razor with tissue aligned longitudinally. Cooling the Tyrode's solution to room temperature caused the PIPAAm to dissolve, releasing the MTF from the cover slip into solution.

For cardiac MTFs studies, individual MTFs were transferred to a $35 \mathrm{~mm}$ Petri dish filled with Tyrodes solution in a custom-built organ bath system (Fig. 1A). The MTFs were mounted in a PDMS clamp $(\sim 5 \times 5 \times 10 \mathrm{~mm})$ to firmly hold them in place during rapid movement. Parallel platinum wire electrodes spaced $\sim 1 \mathrm{~cm}$ apart were lowered directly into the Petri dish. Digital video of MTF contractions was recorded and an external field stimulator (Myopacer, IonOptix Corp.) was used to apply a $10 \mathrm{msec}$ duration square waves between the electrodes at $0.5 \mathrm{~Hz}$ at a voltage $10 \%$ greater than that required to induce contraction. The digital video was converted to a binary skeleton representation using ImageJ and then loaded into MATLAB (Mathworks, Inc.) where the average radius of curvature was found and used to calculate the stress generated by the engineered myocardium for each video frame (Fig. 1B-D, Movie S1). Spontaneous contractions, usually initiated at the free end of the cantilever, and propagation in anisotropic cardiac MTFs were tracked using image processing code written in MATLAB. Briefly, a spline curve was generated for each video frame of the contracting MTF and the radius of curvature for each point along the MTF was calculated. The position of maximum curvature (smallest radius of curvature) was identified and considered to be the location of the mechanical wave front. Velocity of the mechanical wave was determined as the distance traveled along the MTF from initiation of contraction until the wave stopped propagating.

For vascular MTF studies, multiple MTFs were transferred to a custom designed $35 \mathrm{~mm}$ Petri dish (Fig. 1E) filled with Tyrode's solution. The Petri dish was fitted with eight stainless steel posts coated with polytetrafluoroethylene (PTFE) to which the MTFs adhered via hydrophobic interactions (Fig. 1F). In this setup, up to eight MTFs can be observed concurrently. Films were arranged such that, when viewed from above, the film appears as the arc of a circular sector. Here, because the PDMS layer was doped with fluorospheres, the MTFs could be observed using a Zeiss Lumar stereomicroscope (Carl Zeiss) in both brightfield and fluorescence. The Tyrodes solution was maintained at $37^{\circ} \mathrm{C}$ for the duration of the experiment. Vascular MTFs were treated with endothelin-1 (ET-1), a natural vasoconstrictor [18], and HA-1077, a rho-kinase inhibitor [19], to demonstrate that engineered VSM tissue recapitulates natural muscle function. Following the experiment, the fluorescent images were postprocessed 
using MATLAB.) to isolate the MTFs and the thresholded images of the MTFs were fit with circles, indicating their radii of curvature.

\subsection{Mechanical Analysis of MTF bending}

MTF bending during muscle contraction (i.e., the change in curvature of the MTF) acts as a readout and correlates directly to the mean cross-sectional stress generated by the cells. To calculate this stress, we consider the MTF a two-layer plane strain beam. One layer is made up of the passive PDMS, while the other is made up of cells that undergo contraction based on the method for finite volumetric growth of Rodriguez, et al. [20], as modified by Ramasubramanian, et al. [21] This method assumes that if the muscle cell and PDMS layers of the thin film were decoupled (Fig. 2), the cells would undergo a stress-free deformation, characterized by shortening along their long axes, $\lambda_{\mathrm{a}}$. When attached to the PDMS substrate, this contraction results in cell stress and substrate bending. We calculate the value of $\lambda_{\mathrm{a}}$ that is necessary to produce a stress sufficient to bend the MTF to the observed curvature.

2.5.1 Radius of Curvature-For the vascular MTF post assay, the radius of curvature $\left(r_{m}\right)$ of the MTF is found from the circular fit. For the cardiac MTF clamp assay, the films do not always have a uniform radius of curvature, so we fit a $2^{\text {nd }}$ order polynomial to the skeletonized image such that

$$
\theta(s)=\alpha s^{2}+\beta s+\gamma
$$

best fits the geometry, where $\alpha, \beta$ and $\gamma$ are constants. Local curvature is taken as $\kappa(s)=\frac{\partial \theta}{\partial s}=2 \alpha s+\beta$. The measured radius of curvature $\left(\mathrm{r}_{\mathrm{m}}\right)$ is taken as the inverse of the average curvature of the fit along the film's length,

2.5.2 Deformation-We assume that the MTF is a two-layer beam that undergoes shear free bending due to contraction of one layer. The uncontracted stress free configuration is assumed to be a straight beam; the deformed configuration is a sector of a cylinder (Fig. 2A). The deformed geometry is given as

$$
r=r(Y), \theta=k Y, z=\lambda Z
$$

where $k$ and $\lambda$ are constants. The total deformation gradient tensor is given by

$$
\begin{aligned}
\mathbf{F}=\left[\begin{array}{ccc}
\lambda_{r} & 0 & 0 \\
0 & \lambda_{\theta} & 0 \\
0 & 0 & \lambda_{z}
\end{array}\right] \\
=\left[\begin{array}{ccc}
\frac{\partial r}{\partial Y} & 0 & 0 \\
0 & k r & 0 \\
0 & 0 & \lambda_{z}
\end{array}\right] .
\end{aligned}
$$

We let both the cell and PDMS layers be incompressible, i.e. the volume ratio, $J=\operatorname{det}(\mathbf{F})=$ $\lambda_{r} \lambda_{\theta} \lambda_{z}=1$, so

$$
r^{2}=\frac{2}{k \lambda} Y+C
$$

where $C$ is a constant. Since $r\left(R_{i}\right)=r_{i}, r\left(R_{o}\right)=r_{o}$, it follows that 


$$
k \lambda=\frac{2\left(R_{i}-R_{o}\right)}{r_{i}^{2}-r_{o}^{2}}, C=\frac{R_{i} r_{o}^{2}-R_{o} r_{i}^{2}}{R_{i}-R_{o}}
$$

We assume that our system is plane strain, i.e. $\lambda_{\mathrm{z}}=1$, so substituting Eqs.(5) and (4) into Eq.

(3) yields the total deformation gradient tensor $\mathbf{F}=\operatorname{diag}\left(\frac{1}{k r}, k r, 1\right)$. This is the deformation that is measured optically in the MTF assay.

2.5.3 Contraction-Stress is calculated as a function of the elastic deformation $\left(\mathbf{F}_{\mathrm{e}}=\mathrm{diag}\right.$ $\left.\left(\lambda_{\mathrm{er}}, \lambda_{\mathrm{e} \theta}, \lambda_{\mathrm{ez}}\right)\right)$, defined as (Fig. 2B)

$$
\mathbf{F}=\mathbf{F}_{\mathbf{e}} \cdot \mathbf{F}_{\mathbf{a}}
$$

where the active deformation is defined as $\mathbf{F}_{\mathbf{a}}=\operatorname{diag}\left(\lambda_{a}^{-1}, \lambda_{a}, 1\right)$ for the muscle layer, where $\lambda_{\mathbf{a}}$ is the deformation of stress free contraction, and $\mathbf{F}_{\mathrm{a}}=\mathbf{I}$ for the PDMS. So, if the two layers were decoupled, the cells would shorten axially by a stretch ratio of $\lambda_{\mathrm{a}}$, while thickening radially by a stretch ratio of $\lambda_{\mathrm{a}}{ }^{-1}$, and the PDMS layer would not deform (Fig. 2B).

2.5.4 Constitutive Laws-Cauchy stress is a function of elastic deformation and is defined as

$$
\sigma_{i}=\lambda_{e i} \frac{\partial w}{\partial \lambda_{e i}}-p
$$

where $i=r, \theta, z, W$ is the strain energy density function, and $p$ is a Lagrange multiplier. Both the PDMS and cell layers are considered Neo-Hookean materials with strain energy density functions in the form

$$
\begin{aligned}
W=\frac{\mu}{2}\left(I_{e 1}-3\right) \\
\quad=\frac{\mu}{2}\left(\lambda_{e r}^{2}+\lambda_{e \theta}^{2}+\lambda_{e z}^{2}-3\right)
\end{aligned}
$$

where $I_{e l}$ is the first strain invariant of the elastic deformation and $\mu$ is the shear modulus, which in an incompressible material is equal to $(1 / 3)^{*}$ Young's Modulus. Here we use a simple strain energy density function. However, more complex forms can be used to capture the change in apparent modulus with increased contraction [22]. The stress generated in the cell layer is the key value obtained from this assay and can be compared directly across samples.

2.5.5 Mechanical Equilibrium-The deformed MTF is in radial and moment equilibrium, given by

$$
\frac{\partial \sigma_{r}}{\partial r}+\frac{\sigma_{r}-\sigma_{\theta}}{r}=0,
$$




$$
\int_{r_{i}}^{r_{o}} \sigma_{\theta} r d r=0
$$

where the boundary conditions are $\sigma_{\mathrm{r}}\left(r_{i}\right)=\sigma_{\mathrm{r}}\left(r_{o}\right)=0$.

The measured radius of curvature $r_{m}$ (Eq.(3)) is assumed to be the midline of the MTF, such that

$$
r_{i}=r_{m}-\frac{h}{2}, \quad r_{o}=r_{m}+\frac{h}{2}
$$

where $h$ is the deformed thickness of the MTF.

After substitution, this leaves two equations, Eqs (9) and (10), and two unknowns, $\lambda_{\mathrm{a}}$ and $h$. The beam thickness was discretized into twenty transmural points and the equilibrium equations were solved by numerical integration. The unknowns $\left(\lambda_{\mathrm{a}}\right.$ and $\left.h\right)$ were determined by Nelder-Mead minimization, using MATLAB (Mathworks). Stress reported is the mean stress of all radial points between $r_{i}$ and $r_{j}$ (Fig. 2A).

The PDMS layer is a thin beam, whose radius of curvature is much greater than its thickness. So, the true deformation of the cell layer is very small $(<1 \%$ shortening). Therefore, the stress calculated here is equivalent to that of an isometric tension test [23]. At the same time, $\lambda_{a}$ represents the shortening that the muscle would undergo if it was unconstrained. Therefore, if appropriate material parameters are chosen for the cell layer, $\lambda_{\mathrm{a}}$ can be compared to muscle shortening experiments [24].

\subsection{Staining and imaging of cytoskeletal architecture}

PDMS coated cover slips with 2D muscle tissue were fixed and stained at time points concurrent with MTF experiments in order to compare cell structure with contractile function. Tissues were fixed with $4 \%$ paraformaldehyde and $0.25 \%$ Triton X-100 and then fluorescently stained. For the VSMCs, laminin patterning was stained with anti-laminin primary antibody (Sigma) labeled with fluorescently labeled secondary antibodies. Actin fibers and nuclei were stained with phalloidin conjugated Alexa-Fluor 633 (Sigma) and DAPI (Sigma). Samples were imaged with a LSM 5 LIVE laser scanning confocal microscope (Carl Zeiss). For the myocytes, the sarcomeres were visualized by staining the Z-disks with monoclonal mouse anti(sarcomeric $\alpha$-actinin) primary antibody (Sigma) followed by staining with fluorescently labeled secondary antibodies. Samples were then concurrently stained with DAPI (Sigma) and phalloidin conjugated to Alexa-Fluor 488 (Invitrogen).

\section{Results}

\subsection{Construction of Cardiovascular Muscular Thin Films}

Muscular thin films were engineered using both cardiomyocytes (cMTFs) and vascular smooth muscle cells (vMTFs). MTFs are constructed by seeding dissociated muscle cells on a multilayer polymer substrate (Fig. 3A). PIPAAm is spin coated onto a glass coverslip and PDMS is spin coated onto of the PIPAAm layer. The cells are seeded on ECM proteins micropatterned onto the PDMS layer. When the media temperature is lowered below $35^{\circ} \mathrm{C}$, the PIPAAm dissolves and the MTF is released and free floating (Fig. 3A). The radius of curvature of the resulting bilaminate structure is indicative of the stress in the cell layer. 
In order to guide alignment of the cells, lines of extracellular matrix proteins (fibronectin or laminin) were microcontact printed onto the PDMS (Fig. 3B). AFM surface scanning revealed that this ECM pattern is approximately $10-20 \mathrm{nM}$ in thickness (Fig. 3C,D). When cells are cultured on the patterned ECM, they spontaneous organize into a contiguous tissue that is aligned with the patterned matrix lines. In these cells, the long axis of the cell and the nuclear eccentricity parallel the underlying matrix pattern. Fluorescent staining of the fixed tissues reveals that the actin aligns with the underlying matrix in the vascular smooth muscle (Fig. 3E). In cardiac muscle (Fig. 3F) the sarcomeric Z-lines are perpendicular to the matrix. In both cases, the cytoskeletal architecture indicates that the primary axis of contraction is in the longitudinal direction.

\subsection{Cardiac Muscular Thin Films}

Cardiac ventricular muscular thin films were used to measure systolic contraction stress and contractile wave speed in engineered myocardial tissues. The cMTFs were engineered as anisotropic, with uniaxial cellular alignment (Fig. 4A). Imaging of the Z-disks by immunostaining sarcomeric $\alpha$-actinin confirmed that alignment of the myofibrils was also anisotropic (Fig. 4B). The cMTFs were mounted in a bath with a PDMS clamp to firmly hold the cMTF in place during rapid movement and parallel platinum wire electrodes to field stimulate muscle contraction (see Fig. 1). During diastole, the cMTF may have a baseline curvature due to resting tension in the tissue construct (Fig. 4C). During contraction, the radius of curvature decreases dramatically (Fig. 4D), due to cardiomyocyte-generated stress of 13.9 $\mathrm{kPa}$ (Fig. 4E, field stimulated at $0.5 \mathrm{~Hz}$ ). In this set of experiments, three cMTFs built from the ventricular myocytes harvested from two different rat pup litters produced a mean peak systolic stress of $9.2 \pm 3.5 \mathrm{kPa}$.

The cMTF goes through substantial bending and deformation during contraction, however, because the cMTF is a thin beam, the actual shortening of the cardiomyocytes is $<1 \%$, i.e. contraction is isometric. Knowing the stress generated by the cMTF and the elastic modulus of the cardiomyocytes $(\mathrm{E}=\sim 30 \mathrm{kPa})$ [25], the unconstrained shortening was estimated as $25 \%$ at peak systole (Fig. 4F). The accurate measurement of the cell and PDMS layer thicknesses are critical for calculating the contraction stress (Fig. 5A.B) and unconstrained shortening (Fig. $\left.5 \mathrm{~A}^{\prime}, \mathrm{B}^{\prime}\right)$. The elastic modulus of the cell layer, however, has little affect on the calculated stress (Fig. 5C) but does have a significant effect is on the calculation of $\lambda_{\mathrm{a}}$ (Fig. $5 \mathrm{C}^{\prime}$ ), so calculating unconstrained shortening is strongly dependent on accurate cell modulus measurements.

The cMTF system is able to provide an estimate of the contractile wave velocity based on the mechanical deformation of the cMTFs. Tracking the mechanical wave requires that contraction in the cMTF initiates at one end and propagates to the other. Spontaneous contraction of cMTFs often initiated at the free-end and propagated to the base (Fig. 4G, Supplementary Movie S2). Tracking the initial position and propagation of maximum curvature along the cMTF from the initiation of contraction until peak systole (uniform curvature) enabled estimation of contractile wave propagation. In the example shown here, the contractile wave speed was $1.875 \mathrm{~cm} / \mathrm{s}$ (Fig. $4 \mathrm{G}$, white “*”), comparable to the velocity of the mechanical wave reported using phase imaging techniques [26].

\subsection{Vascular Muscular Thin Films}

Vascular muscular thin films were used to demonstrate the potential of this method as a pharmaceutical screening assay. Human umbilical artery VSMCs were engineered as anisotropic monolayers aligned parallel to the long axis of the MTF (Supplementary Movie S3). The vMTFs were adhered to PTFE coated posts via hydrophobic interaction with the PDMS (Fig. 6A). This arrangement allowed multiple vMTFs to be viewed concurrently. Here, eight vMTFs were tested using this assay. The fluorosphere doped PDMS could be viewed 
using fluorescent stereomicroscopy (Fig. 6B,C). In this arrangement, the films are easily approximated as circular arcs (Fig. 6D).

The vMTFs were treated with the endothelium -produced vasoconstrictor endothelin-1 followed by the rho-kinase inhibitor HA-1077, in order to calculate all of the relevant stress states of arterial muscle. The vMTFs had an initial stable baseline curvature (Fig. 6F) indicating that the cells generated a basal stress, defined as the sum of the passive residual stress and the basal contractile tone, of $17.1 \pm 1.7 \mathrm{kPa}$ (Fig. 6G). This value represents the resting tension of the tissue. At time 0 , the vMTFs were stimulated with $50 \mathrm{nMET}-1$, inducing contraction, which caused a decrease in their radii of curvature (Fig. 6F, Supplementary Movie S4) as the cell generated stress increased by $5.06 \pm 0.75 \mathrm{kPa}$ (Fig. 6G). Treatment with $100 \mu \mathrm{M} \mathrm{HA}-1077$, a rho-kinase inhibitor, caused a rapid increase in radius of curvature (Fig. 6F), due to inhibition of contraction (Fig. $6 \mathrm{G}$ ), and resulted in a stress of $3.1 \pm 0.8 \mathrm{kPa}$. The HA-1077 dosage is sufficient to inhibit all myosin light chain phosphorylation [27], so this value represents the residual stress $[22,28]$, or the stress generated by the cytoskeletal elements not involved in the contractile apparatus, but which remains after all other loads are removed. By comparing the residual stress following HA-1077 treatment to the pre ET-1 treated tissue, we can determine that the vMTFs had a basal contractile tone of $13.1 \pm 2.1 \mathrm{kPa}$. This protocol demonstrates that the vMTFs are able to mimic well documented native vascular behavior and implies that this assay could be used to test the effects of pharmaceutical agents on vascular contractility.

\section{Discussion}

Mechanical forces drive numerous cardiovascular processes from morphogenesis during embryonic development [29] to tissue remodeling during adulthood [30]. Maladaptive responses to stresses potentiate a number of cardiovascular diseases, including cardiac hypertrophy [31] and vascular aneurysms [32]. The MTF assay provides a simple method for calculating tissue-level stresses in vitro with a preparation that recapitulates the tissue microenvironment, while avoiding complicating factors inherent to in vivo systems. This approach enables the exploration of the effects of tissue architecture, pharmacologics, and other experimental interventions and allows direct comparison with organ-level contractile function. Moreover, the use of standard optical techniques makes the MTF assay simpler than traditional contractility assays that require force gauges or complex transducers.

Here, we validated our system using engineered cardiovascular muscle tissues from both cardiomyocytes and vascular smooth muscle cells. The cMTFs were engineered to mimic ventricular lamellae. The measured peak systolic stress and constrained shortening are comparable to isometric measurement of isolated papillary muscle [33] (Fig. 4E). Thus, the cMTF system we have developed recapitulates both the anisotropic alignment of normal cardiac muscle and physiologically relevant, systolic stress levels.

A unique aspect of the MTF contractility assay is the capability to track local changes in radius of curvature along its length during the cardiac cycle (Fig. 4G). Previous work demonstrated the coupling of the action potential and contractile waves during spiral wave reentry, suggesting that there is information content in the contractile wave that indicates the degree of coupling between cells in a tissue [34]. Further, we can detect dyssynchronous contraction when the mechanical wave fails to propagate, or initiates at multiple locations at the same time, resulting in a fluttering cMTF with no discernable deflection (Supplementary Movie S5). Thus, a broad range of qualitative and quantitative data can be extracted from the assay by proper frame by frame analysis of the deformation.

The vMTFs were used to mimic the lamellae of the arterial tunica media. The vMTF assay confirms the presence of functional ET-1 receptors in the engineered smooth muscle and is 
able to accurately quantify the magnitude of induced contraction [12]. Concurrent monitoring of eight vMTFs contracting and relaxing (Fig. 6 B-G) demonstrates that this technique produces engineered smooth muscle that repeatability responds to pharmacologic stimulation at physiologic stress levels [35]. Moreover, this assay demonstrates that MTFs can provide a method for studying diseases and potential therapeutic interventions, with the potential to significantly scale up the throughput. As an early screening method, this high-fidelity, in vitro contractility assay could be used to directly test the effect of drugs on contractility and potentially decrease the high failure rate of cardiovascular drugs, as currently, novel molecules reaching Phase 1 clinical trials for cardiovascular drugs have a completion rate of less than $20 \%$ [36].

Similar methods have used a modified Stoney's equation to calculate cardiomyocyte stress [13-15]. The method described here is a marked improvement on this previous approach because Stoney's equation assumes that the cell layer is thin compared to the substrate and undergoes isotropic contraction. Our method is specifically derived to study aligned tissues with orthotropic contraction, which is more representative of in vivo muscle structure. Further, this method makes no assumptions about the relative thicknesses of the layers and is thus equally accurate for a wide range of substrate and tissue thicknesses.

Because MTFs employ a single monolayer of cells, they are not perfusion limited, as are many tissue engineered constructs. Additionally, the similarity of this assay and traditional in-dish 2-D culture means that MTF results can be directly compared to common biological assays done on plated cells. Moreover, MTFs are potentially deployable in vivo therapeutically, meaning that an in vitro construct's physiological quality can be assessed prior to implantation. Finally, the assay could be extended to striated skeletal and pulmonary, bladder or uterine smooth muscles. Further development should enable assessment of non-muscle tissues such as skin, myofibroblast-rich wounds and other epithelial layers such as the cornea, where contractility is an often neglected but critically important component of tissue function.

\section{Conclusions}

Here, we report a method for measuring stress in engineered cardiovascular tissue using biohybrid thin films constructed from muscle cells micropatterned onto thin flexible PDMS sheets. We have also developed an elasticity based analysis for calculating the contraction stress generated by this engineered muscle. This method was validated using both rat cardiac myocytes and human umbilical artery vascular smooth muscle cells. End-systolic contraction stress and contractile wave speed were measured in electrically stimulated cardiomyocyte constructs, while pharmacologically induced contraction and relaxation were measured in the VSMC constructs. These proof-of-concept experiments demonstrate two key advantages of this MTF assay compared to existing approaches. (i) MTFs can be engineered with consistent tissue microstructures that generate repeatable contractile properties. (ii) High-speed, highresolution digital imaging of MTF curvature enables even small forces $(<1 \mathrm{kPa})$ to be measured with excellent signal-to-noise ratios. Additionally, the stress sensitivity of the MTF can cover a wide physiologic range by tuning the substrate thickness. Thus, this assay has potential for broad applicability in any stress-bearing cell type.

\section{Supplementary Material}

Refer to Web version on PubMed Central for supplementary material.

\section{Acknowledgments}

We acknowledge financial support from the DARPA Biomolecular Motors Program and PREVENT program, NIH R01HL079126-01A2, and the Harvard Materials Research Science and Engineering Center (MRSEC). 


\section{REFERENCES}

1. Rohr S, Scholly DM, Kleber AG. Patterned growth of neonatal rat heart cells in culture. Morphological and electrophysiological characterization. Circ Res 1991;68:114-30. [PubMed: 1984856]

2. Gillis AM, Fast VG, Rohr S, Kleber AG. Spatial changes in transmembrane potential during extracellular electrical shocks in cultured monolayers of neonatal rat ventricular myocytes. Circ Res 1996;79:676-90. [PubMed: 8831491]

3. Bursac N, Parker KK, Iravanian S, Tung L. Cardiomyocyte cultures with controlled macroscopic anisotropy - A model for functional electrophysiological studies of cardiac muscle. Circ Res 2002;91:E45-E54. [PubMed: 12480825]

4. Parker KK, Tan J, Chen CS, Tung L. Myofibrillar architecture in engineered cardiac myocytes. Circ Res 2008;103:340-2. [PubMed: 18635822]

5. Entcheva E, Bien H, Yin LH, Chung CY, Farrell M, Kostov Y. Functional cardiac cell constructs on cellulose-based scaffolding. Biomaterials 2004;25:5753-62. [PubMed: 15147821]

6. Thakar RG, Ho F, Huang NF, Liepmann D, Li S. Regulation of vascular smooth muscle cells by micropatterning. Biochem Biophys Res Commun 2003;307:883-90. [PubMed: 12878194]

7. Isenberg BC, Tsuda Y, Williams C, Shimizu T, Yamato M, Okano T, et al. A thermoresponsive, microtextured substrate for cell sheet engineering with defined structural organization. Biomaterials 2008;29:2565-72. [PubMed: 18377979]

8. Eschenhagen T, Fink C, Remmers U, Scholz H, Wattchow J, Weil J, et al. Three-dimensional reconstitution of embryonic cardiomyocytes in a collagen matrix: a new heart muscle model system. FASEB J 1997;11:683-94. [PubMed: 9240969]

9. Baar K, Birla R, Boluyt MO, Borschel GH, Arruda EM, Dennis RG. Self-organization of rat cardiac cells into contractile 3-D cardiac tissue. FASEB J 2004;18:275-7. [PubMed: 15574489]

10. Niklason LE, Gao J, Abbott WM, Hirschi KK, Houser S, Marini R, et al. Functional arteries grown in vitro. Science 1999;284:489-93. [PubMed: 10205057]

11. L'Heureux N, Stoclet J-C, Auger FA, Lagaud GJ-L, Germain L, Andriantsitohaina R. A human tissueengineered vascular media: a new model for pharmacological studies of contractile responses. FASEB J 2001;15:515-24. [PubMed: 11156967]

12. Laflamme K, Roberge CJ, Pouliot S, D'Orleans-Juste P, Auger FA, Germain L. Tissue-engineered human vascular media produced in vitro by the self-assembly approach present functional properties similar to those of their native blood vessels. Tissue Eng 2006;12:2275-81. [PubMed: 16968167]

13. Feinberg AW, Feigel A, Shevkoplyas SS, Sheehy S, Whitesides GM, Parker KK. Muscular thin films for building actuators and powering devices. Science 2007;317:1366-70. [PubMed: 17823347]

14. Kim J, Park J, Na K, Yang S, Baek J, Yoon E, et al. Quantitative evaluation of cardiomyocyte contractility in a 3D microenvironment. J Biomech 2008;41:2396-401. [PubMed: 18644311]

15. Domian IJ, Chiravuri M, van der Meer P, Feinberg AW, Shi X, Shao Y, et al. Generation of functional ventricular heart muscle from mouse ventricular progenitor cells. Science 2009;326:426-9. [PubMed: 19833966]

16. Adams WJPT, Geisse NA, Sheehy S, Parker KK. Engineering design of a cardiac myocyte. J Computer-Aided Mater Des 2007;14:19-29.

17. Han M, Wen J-K, Zheng B, Cheng Y, Zhang C. Serum deprivation results in redifferentiation of human umbilical vascular smooth muscle cells. Am J Physiol Cell Physiol 2006;291:C50-8. [PubMed: 16467401]

18. Yanagisawa M, Inoue A, Ishikawa T, Kasuya Y, Kimura S, Kumagaye S, et al. Primary structure, synthesis, and biological activity of rat endothelin, an endothelium-derived vasoconstrictor peptide. PNAS 1988;85:6964-7. [PubMed: 3045827]

19. Seto M, Sasaki Y, Sasaki Y, Hidaka H. Effects of HA1077, a protein kinase inhibitor, on myosin phosphorylation and tension in smooth muscle. Eur J Pharmacol 1991;195:267-72. [PubMed: 1874276]

20. Rodriguez EK, Hoger A, McCulloch AD. Stress-dependent finite growth in soft elastic tissues. J Biomech 1994;27:455-67. [PubMed: 8188726]

21. Ramasubramanian A, Taber L. Computational modeling of morphogenesis regulated by mechanical feedback. Biomech Model Mechanobiol 2008;7:77-91. [PubMed: 17318485] 
22. Alford PW, Humphrey JD, Taber LA. Growth and remodeling in a thick-walled artery model: effects of spatial variations in wall constituents. Biomech Model Mechanobiol 2008;7:245-62. [PubMed: 17786493]

23. Layland J, Young IS, Altringham JD. The effect of cycle frequency on the power output of rat papillary-muscles in-vitro. J Exp Biol 1995;198:1035-43. [PubMed: 7730751]

24. Chemla D, Lecarpentier Y, Martin JL, Clergue M, Antonetti A, Hatt PY. Relationship between inotropy and relaxation in rat myocardium. Am J Physiol 1986;250:H1008-16. [PubMed: 3717355]

25. Lieber SC, Aubry N, Pain J, Diaz G, Kim SJ, Vatner SF. Aging increases stiffness of cardiac myocytes measured by atomic force microscopy nanoindentation. Am J Physiol Heart Circ Physiol 2004;287:H645-H51. [PubMed: 15044193]

26. Hwang, S-m; Yea, K-h; Lee, KJ. Regular and alternant spiral waves of contractile motion on rat ventricle cell cultures. Phys Rev Lett 2004;92:198103. [PubMed: 15169449]

27. Nagumo H, Sasaki Y, Ono Y, Okamoto H, Seto M, Takuwa Y. Rho kinase inhibitor HA-1077 prevents Rho-mediated myosin phosphatase inhibition in smooth muscle cells. Am J Physiol Cell Physiol 2000;278:C57-65. [PubMed: 10644512]

28. Fung YC, Liu SQ. Change of residual strains in arteries due to hypertrophy caused by aortic constriction. Circ Res 1989;65:1340-9. [PubMed: 2805247]

29. Voronov DA, Alford PW, Xu G, Taber LA. The role of mechanical forces in dextral rotation during cardiac looping in the chick embryo. Dev Biol 2004;272:339-50. [PubMed: 15282152]

30. Cowin SC. Tissue growth and remodeling. Annu Rev Biomed Eng 2004;6:77-107. [PubMed: 15255763]

31. Ruwhof C, van der Laarse A. Mechanical stress-induced cardiac hypertrophy: mechanisms and signal transduction pathways. Cardiovasc Res 2000;47:23-37. [PubMed: 10869527]

32. Baek S, Rajagopal KR, Humphrey JD. A Theoretical model of enlarging intracranial fusiform aneurysms. J Biomech Eng 2006;128:142-9. [PubMed: 16532628]

33. Nishimura S, Yasuda S, Katoh M, Yamada KP, Yamashita H, Saeki Y, et al. Single cell mechanics of rat cardiomyocytes under isometric, unloaded, and physiologically loaded conditions. Am J Physiol Heart Circ Physiol 2004;287:H196-H202. [PubMed: 15001443]

34. Bers DM. Cardiac excitation-contraction coupling. Nature 2002;415:198-205. [PubMed: 11805843]

35. Cox RH. Arterial wall mechanics and composition and the effects of smooth muscle activation. Am J Physiol 1975;229:807-12. [PubMed: 1211473]

36. Bril A, Avkiran M. Translating molecular target into drug therapy in cardiovascular disease. Curr Opin Pharmacol 2008;8:111-3. [PubMed: 18294914] 


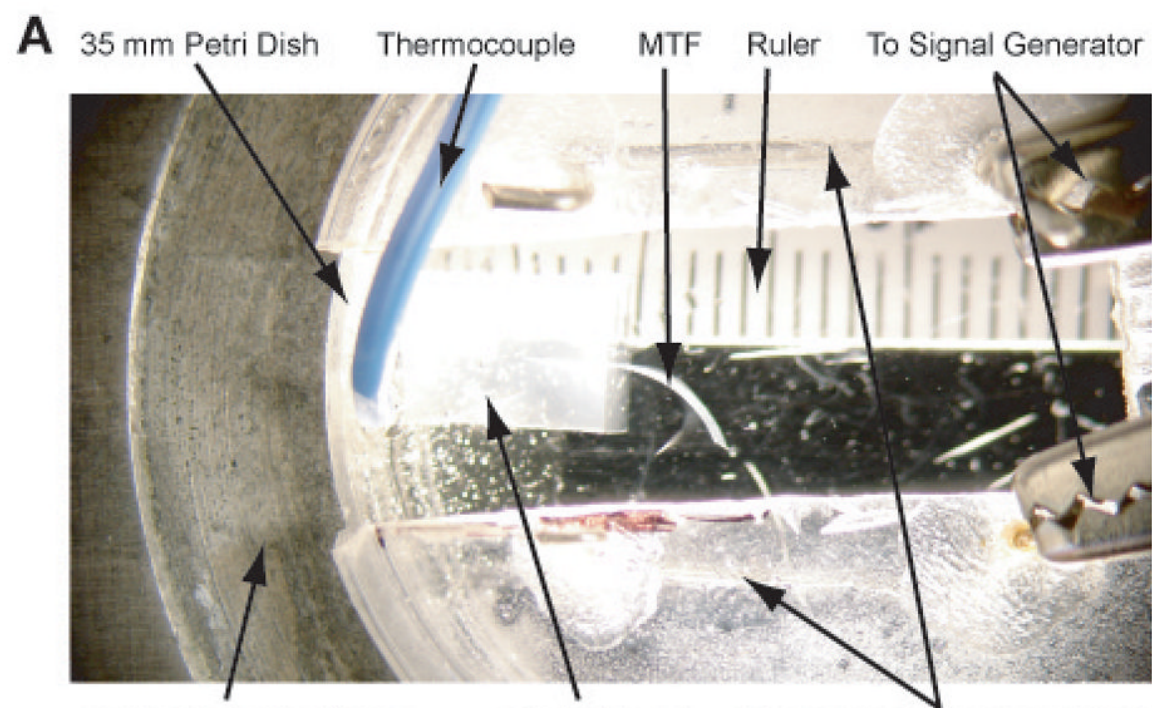

Heated Aluminum Stage

B

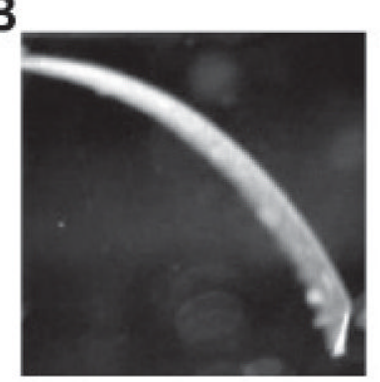

PDMS Clamp

C

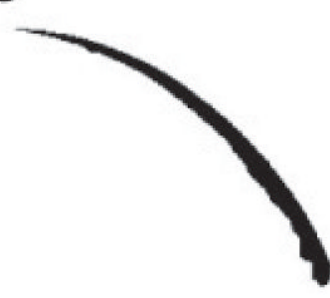

D

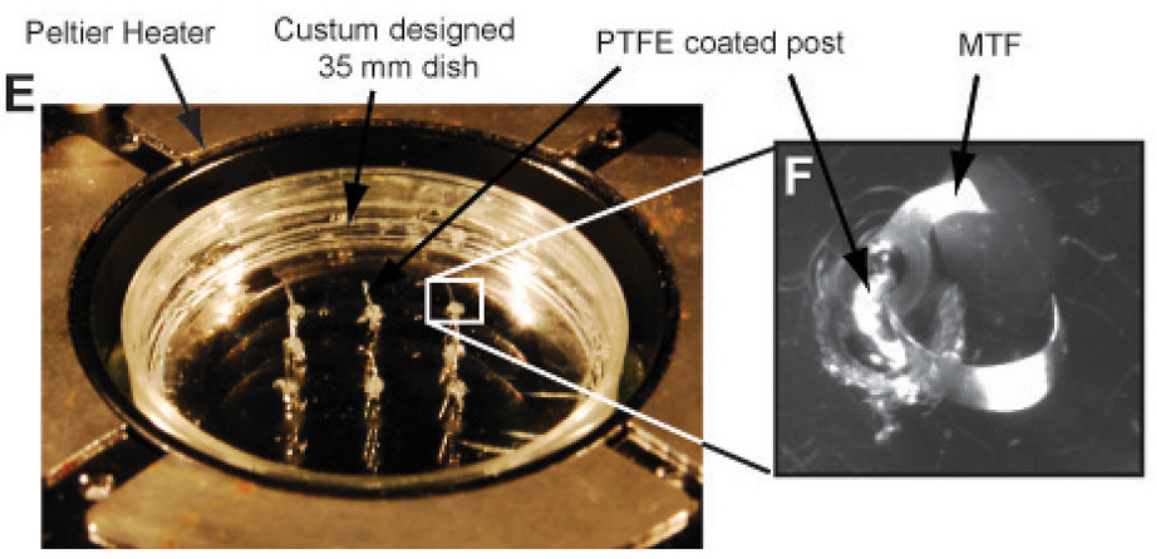

Figure 1.

(A) Image of the experimental setup used to measure cMTF contractions. The cMTF is mounted sideways in a PDMS clamp that is fixed to the bottom of a $35 \mathrm{~mm}$ diameter Petri dish. A signal generator is attached to the parallel platinum electrodes to provide controlled pacing signals. A millimeter scale ruler is placed below the Petri dish as a calibration reference. The entire setup is placed on a stereomicroscope with darkfield illumination. (B-D) Representative steps of the image processing procedure used to determine the MTF radius of curvature during contraction. (B) The raw grayscale image is digital recorded. (C) The image is thresholded to convert it to a binary image. (D) The binary image is skeletonized to find the midline. (E-F) Images of experimental setup used to measure vMTF contractions. (E) vMTFs are imaged in 
a custom designed $35 \mathrm{~mm}$ dish. Eight PTFE posts are arranged so they stand vertically within the dish. Dish is heated with a Peltier heater during the experiment. (F) One MTF is mounted on each post. 
A
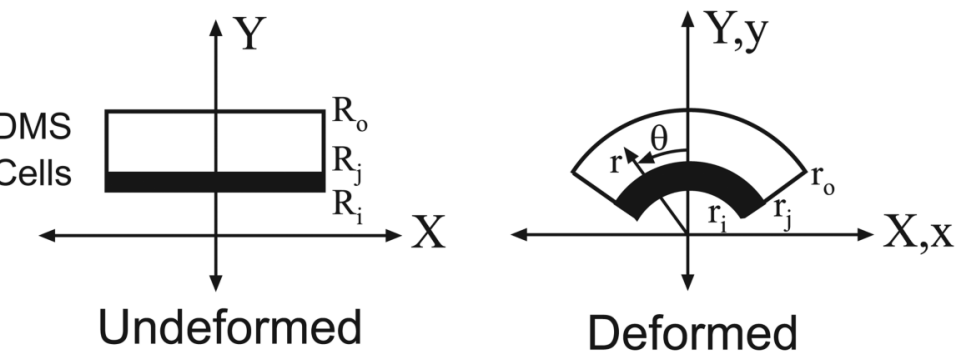

B
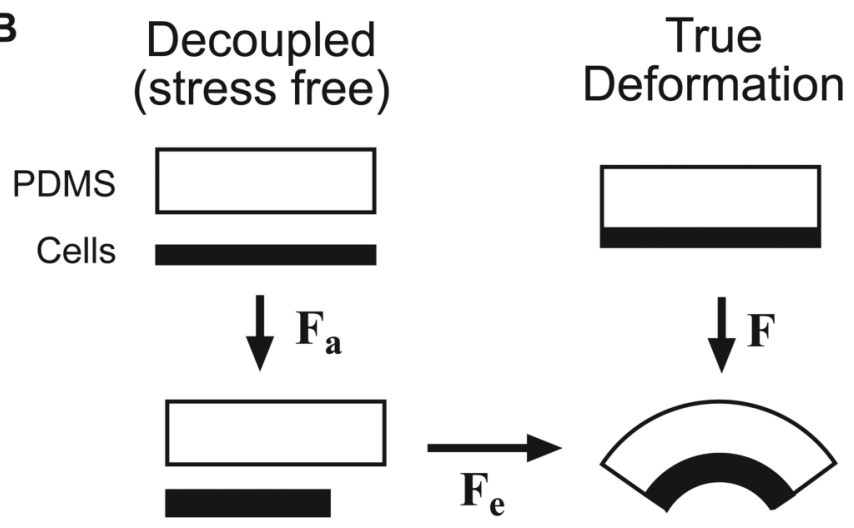

Figure 2.

Schematic representation of analysis method. (A) The beam is assumed to be straight and stress free in the undeformed configuration. In the deformed configuration, the beam is assumed to have a uniform curvature. (B) The analysis assumes that if the PDMS layer and cell layer are decoupled the cell layer will undergo a stress free deformation described by $\mathbf{F}_{\mathbf{a}}$. The true, measured, deformation is given by $\mathbf{F}$. The elastic deformation is given by $\mathbf{F}_{\mathbf{e}}$ and is defined as $\mathbf{F}_{\mathbf{e}}=\mathbf{F} \cdot \mathbf{F}_{\mathbf{a}}$ 

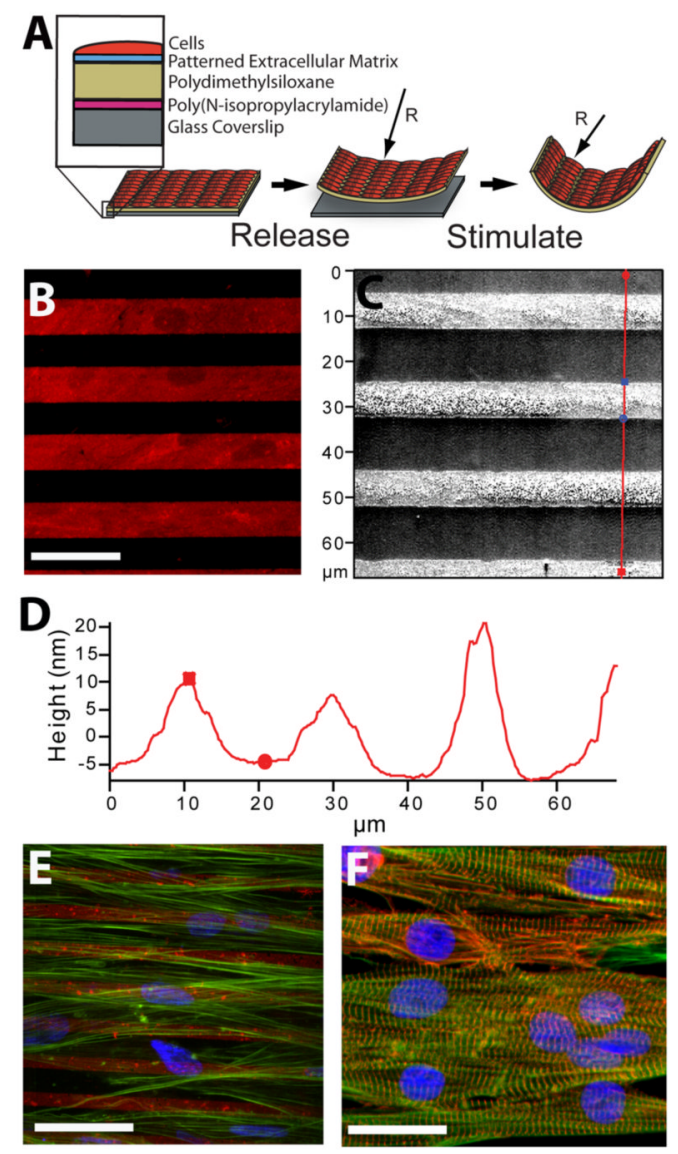

Figure 3.

Muscular thin film method and microstructure. (A) Schematic representation of MTF assay illustrating MTF fabrication, release and the change in radius of curvature during muscle contraction. (B) Immunofluorescent image of $20 \mu \mathrm{m}$ lines of laminin spaced by $20 \mu \mathrm{m}$ gaps. (scale: $50 \mu \mathrm{m})(\mathrm{C})$ Topological scan of $10 \times 10 \mu \mathrm{m}$ stamped laminin, using atomic force microscope. (D) Line scan following line in red in (C) showing the height change of substrate following LAM stamping. (E) Anisotropically patterned vascular smooth muscle tissue; red: $10 \times 10 \mu \mathrm{m}$ patterned LAM, green: Factin, blue: nuclei. (scale: $50 \mu \mathrm{m})(\mathbf{F})$ Anisotropically patterned cardiomyocytes; red: $\alpha$-actinin, green: F-actin, blue: nuclei. (scale: $30 \mu \mathrm{m}$ ) 

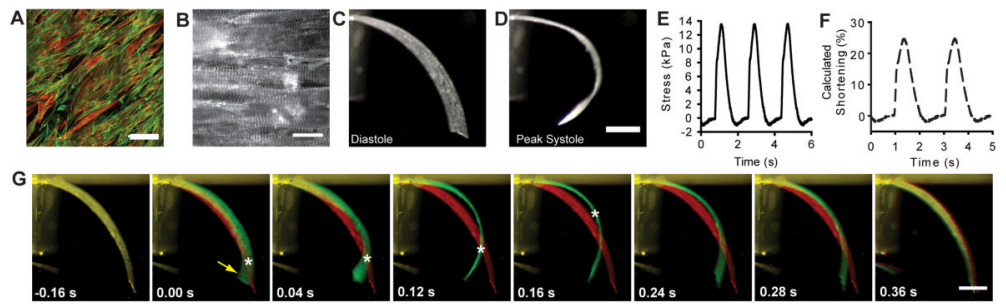

Figure 4.

Analysis of microscale cytoskeletal structure and macroscale contractility of anisotropic cardiac MTFs. (A) Confocal image of an engineered anisotropic cardiac monolayer stained for F-actin (green) and sarcomeric $\alpha$-actinin (red). (B) Close-up of anisotropic monolayer showing uniaxial orientation of sarcomere Z-disks (stained for sarcomeric $\alpha$-actinin). Example anisotropic cardiac MTF in (C) diastole and (D) peak systole during $0.5 \mathrm{~Hz}$ paced contraction. (E) Stress versus time for an anisotropic cardiac MTF during $0.5 \mathrm{~Hz}$ paced contractions. (F) The anisotropic cardiac MTF undergoes isometric contraction shortening $<1 \%$ at peak systole, however knowing the stress generated by the muscle and the elastic modulus of the cells (E $\sim 30 \mathrm{kPa}$ ) the unconstrained shortening can be estimated as $\sim 25 \%$ at peak systole (dashed line, see Online Methods for details). (G) Velocity of the contractile wave can be calculated from the spontaneous contraction of an anisotropic cardiac MTF from diastole at $\mathrm{t}=-0.16 \mathrm{~s}$ (red) and observing deformation at subsequent time points (green) from the initiation of contraction at the free end of the MTF (yellow arrow, $t=0)$ that propagates to the base of the MTF $(t=$ $0.16 \mathrm{~s})$ and then relaxes back to it diastolic state $(\mathrm{t}=0.36 \mathrm{~s})$. Image processing of the sequence in to track the location of the contractile wave (white ' $*$ ') based on the position of maximum curvature (smallest radius of curvature). Scale bars are (A) $100 \mu \mathrm{m}$, (B) $20 \mu \mathrm{m}$ and (C,D, and G) $1 \mathrm{~mm}$. 

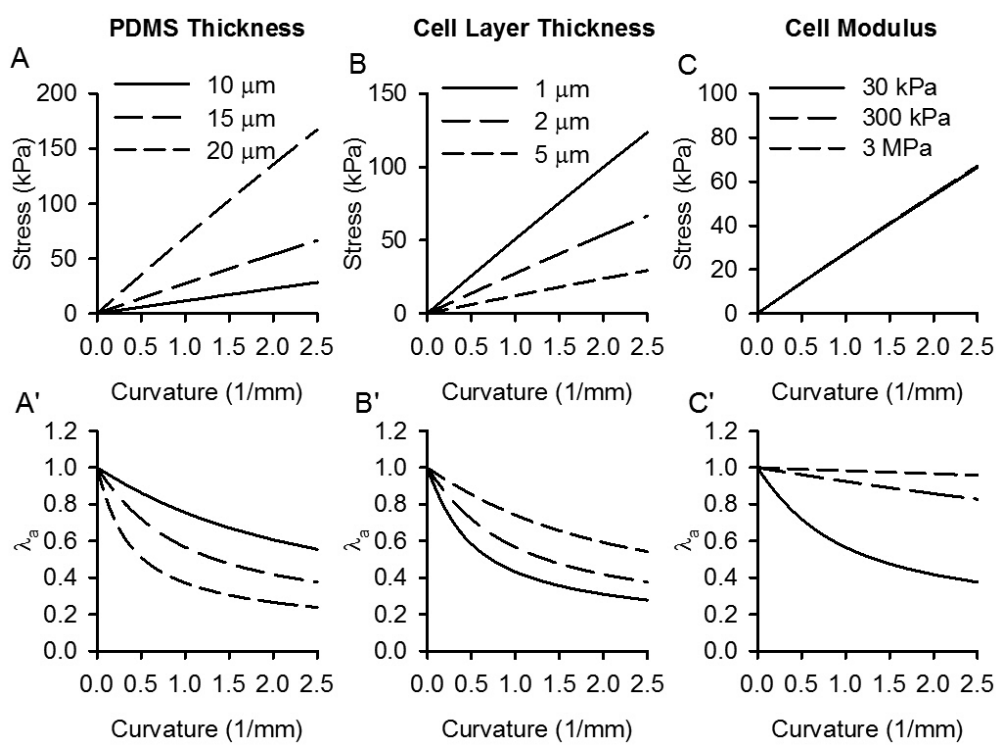

Figure 5.

Analysis parameter study. (A,B,C) Calculated stress for a given observed curvature for varying PDMS thickness, cell layer thickness and cell modulus. $\left(\mathbf{A}^{\prime}, \mathbf{B}^{\prime}, \mathbf{C}^{\prime}\right)$ For the same parameter variations, the stress-free shortening of the cell layer necessary to generate the observed curvature. Note that cell modulus has virtually no affect on cell stress but has a large affect on stress-free shortening. 

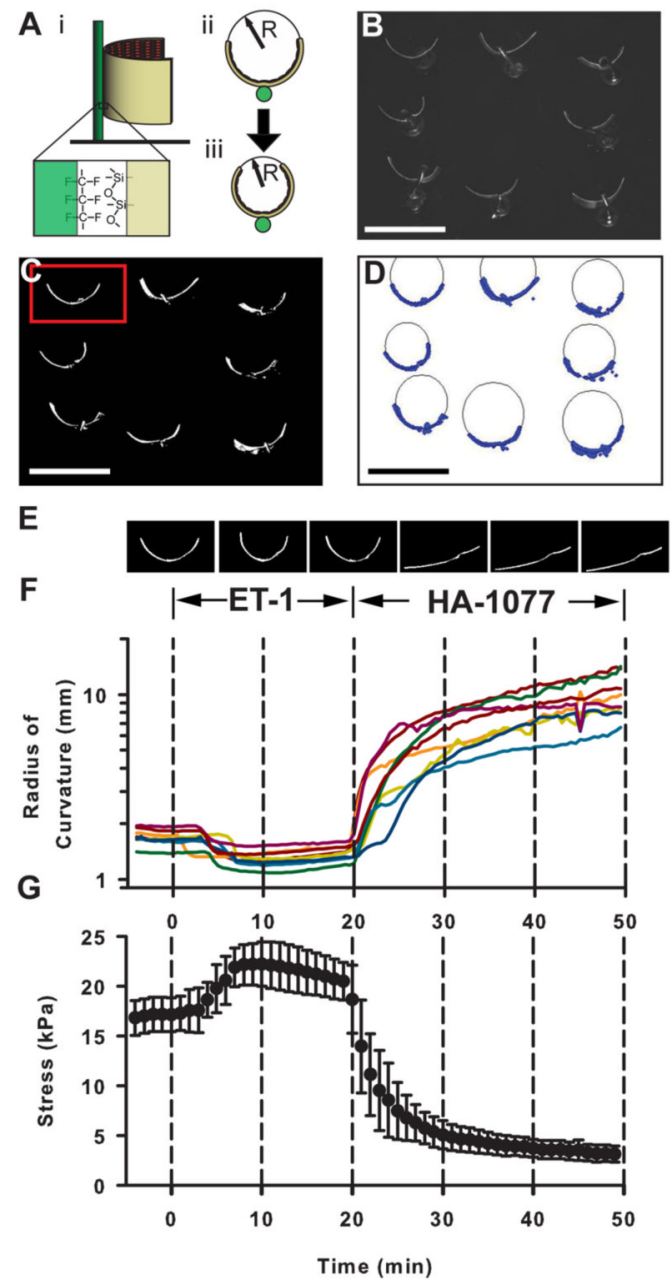

Figure 6.

Multi-film assay for measurement of vascular smooth muscle contraction. (A) Schematic representation of vMTF assay. (i) MTFs are adhered to PTFE coated posts by hydrophobic interaction with the PDMS. (ii,iii) Radius of curvature of each film can be observed from above. (B) Fluorescent image of eight vMTFs mounted in custom designed petri dish. (C) Thresholded image of vMTFs. (D) Circular fit to thresholded image. (E-G) Films were exposed to $50 \mathrm{nM}$ ET-1 for twenty minutes, starting at time zero. After twenty minutes, $100 \mu \mathrm{M} \mathrm{HA-1077}$ was added to the solution. (E) Thresholded images of temporal progression of the MTF highlighted in (C). (F) Radii of curvature for the eight vMTFs in (B-D) during treatment. (G) Stress in muscle layer for all vMTFs during the experiment (mean \pm standard deviation). 